\title{
The role of antimicrobial peptides in the prevention of sexually transmitted infection (STI)
}

\begin{abstract}
Development of sexually transmitted infections (STI) has existed in human societies along the history of mankind. It is still an important issue and research to diagnose and treat this group of diseases is growing. Due to the microbial antibiotic resistance to classical antibiotics, finding new antimicrobial compounds is essential. Aantimicrobial peptides (AMPs) are of the most important of these compounds. AMPs ususally show a broad spectrum of activities. Defensins, secretory leukocyte protease inhibitors (SLPI), calprotectin, lysozyme, lactoferrin and elafin are familes of antimicrobial peptides that are involved in vaginal innate immunity. Some of AMPs such as LL-37, magainin 2 and nisin A have dual spermicide and microbicide effects. LL-37 is the most suitable peptide for this purpose with both spermicide/microbicide properties. LL-37 formulated gel administered into the vagina would exert microbicidal action on uropathogenic microbes.However, LL-37 is a long peptide and its synthesis is very expensive, hence the shortest truncated active form of LL-37 peptide or its mimetic, has to be designed. Also, setting up production method for recombinant LL-37 is necessary. In addition in silico methods can help this process and modification of AMPs. So, the proper formulation of the LL-37 as a vaginal spermicide/microbicide can play a role in the health of women.
\end{abstract}

Keywords: antimicrobial peptide, STI, defensin, LL-37, spermicide
Volume 8 Issue 2 - 2019

\author{
Hamid Madanchi, Sorush Sardari \\ Department and Center for Biotechnology Research, Semnan \\ University of Medical Sciences, Semnan, Iran
}

\section{Correspondence: Soroush Sardari, Drug Design and} Bioinformatics Unit, Department of Medical Biotechnology, Biotechnology Research Center, Pasteur Institute of Iran, Tehran | 3164, Iran, Tel/Fax (+98-2I) 66480780, Mobile (+98-912) 2632484; Email ssardari@hotmail.com

Received: July 22, 2017 | Published: April 03, 2018

\section{Introduction}

Sexually Transmitted Infections (STIs) such as Chlamydia infection, chancroid, gonorrhea, crabs (Pubic Lice), genital herpes, hepatitis B, HIV/AIDS, Human Papillomavirus (HPV), scabies, trichomoniasis and Molluscum Contagiosum (MCV) also known as Sexually Transmitted Diseases (STDs) or Venereal Diseases (VD)[1]. STIs are diseases that are passed on from one person to another through sexual contact, and sometimes by genital contact - the infection can be passed on via vaginal intercourse, oral sex, and anal sex. ${ }^{1}$ Development of sexually transmitted infections (STI) in human societies, caused that research on diagnosise and treatment of these diseases is growing. Despite successes in antimicrobial drug development, resistance to every new antibiotic molecule has appeared in bacterial populations within a few years of its introduction determining a reduction in the therapeutic efficacy. Aantimicrobial peptides (AMPs) have received an increasing attention as potential therapeutic agents. ${ }^{2}$ AMPs are important components of innate immune systems. On one hand, there is an urgent demand for novel antimicrobials due to the current trend of reduction in the potency of commonly used antibiotics. ${ }^{3}$ AMPs show a broad spectrum of activities against gram-negative and gram-positive bacteria, including antibiotic-resistant bacterial strains and some fungi, viruses, and parasites. ${ }^{4}$ Also some of them have spermicidal properties. ${ }^{4}$ Many of these peptides such as natural vaginal peptides and synthetic spermicide and microbicide to prevention and treatment of sexually transmitted diseases can be investigated. In this review, we introduce some of the most important AMPs in this regard.

\section{Antimicrobial peptides that are involved in vaginal innate immunity}

Defensins, secretory leukocyte protease inhibitors (SLPI), calprotectin, lysozyme, lactoferrin and elafin are familes of antimicrobial peptides that are involved in vaginal innate immunity. ${ }^{5}$
Both $\alpha$ - and $\beta$-defensins have been shown to have anti-HIV activity. $\alpha$-defensins directly inhibit viral replication. ${ }^{5,8} \beta$-defensins have also been shown to decrease the expression of coreceptors required for virus entry into the host cell. ${ }^{5,9}$ Some of these AMPs, can regulate the level of sex hormones. Concentrations of $\alpha$ - and $\beta$-defensins from cervicovaginal lavage can vary greatly throughout the menstrual cycle, a variation of up to 50 -folds. ${ }^{5,10}$ This change in concentration is evidence that levels of estrogen can influence the operational status of the vaginal innate immune system. ${ }^{5,11}$ SLPI has also been shown to inhibit HIV-1 activity[9]. Reduced SLPI levels have also been found in women with lower genital tract infections from Trichomonas vaginalis, C. trachomatis, N. gonorrhoeae and Candida. ${ }^{11}$ Levels of calprotectin also correlate positively with inflammatory cytokines in cervical mucus, indicating that it plays a role in local inflammation. Also, calprotectin have been shown to have anti-Candida activity. ${ }^{5,12}$ Elafin like defencin and SLPI has anti-HIV activity. In vivo studies have shown that elafin can cause resistance to HIV infection. ${ }^{13}$

\section{Spermicide and microbicide peptides}

Some of AMPs such as LL-37, maximin 1, maximin3, magainin 2, dermaseptin-S1, dermaseptin-S4, subtilosin A, pediocin PA-1/ AcH, nisin A, lacticin 3147, sarcotoxin Pd and gramicidin A have dual spermicide/microbicide activity (Table 1). LL-37, magainin 2 and nisin A are the only AMPs with confirmed in vivo contraceptive effects in experimental animals. ${ }^{14,15}$ The acidic $\mathrm{pH}$ in the healthy human vagina is attributed to the presence of commensal lactobacilli. nisin A is not a good candidate to be developed into a vaginal microbicide, since it exerts microbicidal activity on vaginal Lactobacillus spp. ${ }^{15}$ LL-37 is produced by cervicovaginal epithelial cells naturally, however, this question arises that can it cause infertility? Therefore, it seems that high concentration of natural LL-37 may cause infertility by spermicide effects. What factors can increase naturally production of LL-37 by cervicovaginal epithelial cells? 
Table I sequence and source of spermicide and microbicidal Peptides (Retrieved from Antimicrobial peptide data base. ${ }^{16}$

\begin{tabular}{lll}
\hline Peptide name & Sequence & Source \\
\hline Maximin I & Gigtkilggvktalkgalkelastyan & Bombina maxima \\
Maximin 3 & Giggkilsglktalkgaakelastylh & Bombina maxima \\
Magainin 2 & Gigkflhsakkfgkafvgeimns & Xenopus laevis \\
Dermaseptin-SI & Alwktmlkklgtmalhagkaalgaaadtisqgtq & Phyllomedusa sauvagii \\
Dermaseptin-S4 & Alwmtllkkvlkaaakaalnavlvgana & Phyllomedusa sauvagii \\
Nisin A & Itsislctpgcktgalmgcnmktatchcsihvsk & Streptococcus lactis \\
LL-37 & Llgdffrkskekigkefkrivqrikdflrnlvprtes & Homo sapiens \\
Gramicidin A & Vgalavvvwlwlwlw & Bacillus brevis \\
PediocinPA-I/AcH & Kyygngvtcgkhscsvdwgkattciinngamawatgghqgnhkc & Pediococcus acidilactici \\
Subtilosin A & Nkgcatcsigaaclvdgpipdfeiagatglfglwg & Bacillus subtilis \\
Lacticin 3I47 & Cstntfslsdywgnngawctlthecmawck & Lactococcus lactis \\
sarcotoxin Pd & Gwlkkigkkiervgqhtrglgiaqiaanvaatar & Paederus dermatitis \\
\hline
\end{tabular}

\section{Discussion}

Bacteria, fungi, yeasts, viruses and protozoa cause sexually transmitted infection. There are many antimicrobial peptides against viruses, bacteria, fungi and protozoa. Several of AMPs have direct anti microbial activity and therefore protect the host from pathogens. Others serve as chemotactic agents, thus recruiting inflammatory cells to the site of infection. ${ }^{5}$ Although some natural AMPs are already in clinical and commercial use, ${ }^{2,6}$ the future design and optimization of novel AMPs will need to improve their antimicrobial activity and stability, minimize the cytotoxicity, immunogenicity and reduce the proteolytic degradation or the biological fluid inhibition. ${ }^{2,7}$ Synthesis of antimicrobial peptides are known to be expensive and time-consuming, therefore insilico methods to AMPs design can reduce production costs and the time required for evaluation of activity and toxicity. ${ }^{2}$ LL37 as a spermicide and microbicide peptidehas antimicrobial effect against Chlamydia trachomatis, Neisseria gonorrhoeae, Treponema pallidum, Candida albicans, Staphylococcus aureus, Pseudomonas aeruginosa, Prevotella intermedia, Porphyromonas gingivalis, Klebsiella pneumoniae, Escherichia coli, Enterococcus faecalis and HIV and HSV. ${ }^{15}$ Therefore, LL-37 exerts microbicidal effects on most of microorganisms that involved in STIs, vaginitis and urinary Tract Infection. ${ }^{15}$ Also, LL-37 completely inhibits human and mouse sperm motility within $5 \mathrm{~min}$ at $10.8 \mu \mathrm{M}$ and $3.7 \mu \mathrm{M}$, respectively. This inhibition is likely due to the specific disruptive effects of LL-37 on sperm surface membranes. ${ }^{15}$ LL-37 formulated gel administered into the vagina would exert microbicidal action on uropathogenic microbes that opportunistically form a reservoir in the vagina ${ }^{15}$ The vaginal secretion, which can travel upwards into the urinary tract, would also likely contain LL-37 released from the gel, which then can fight against microbes in this tract. ${ }^{15}$

\section{Conclusion}

As mentioned above, the STIs are inhibited by AMPs. LL-37 is the most suitable peptide for this purpose with both spermicide/ microbicide properties.LL-37 is a long peptide and its synthesis is very expensive, so the shortest truncated LL-37 peptide or its mimetic, which still bears spermicidal and microbicidal activity, has to be discovered. Also, it is necessary to produce recombinant LL-37.
In silico methods can help this process. On the other hand, an effective method to deliver LL-37 into the vaginal lumen has to be established. ${ }^{15}$ with further studies; modificationsand changes in AMPs such as LL37 can overcome the limitations of their use. Eventually, modified AMPs can be formulated in the form of ointments and topical lotions and play an important role in the health of women.

\section{Acknowledgments}

None.

\section{Conflicts of interest}

The author declares there are no conflicts of interest.

\section{References}

1. Nordqvist Christian. Sexually Transmitted Infections (STIs): Types, Symptoms. Medical News Today. Medil exicon. 2015.

2. G Maccari R Nifosì, M Di Luca. Rational development of antimicrobial peptides for therapeutic use: design and production of highly active compounds. Microbial pathogens and strategies for combating them: science, technology and education (A. Méndez-Vilas, Ed.). 2013;12651277.

3. Guangshun Wang, Biswajit Mishra, Kyle Lau, et al. Antimicrobial Peptides in 2014. Pharmaceuticals (Basel). 2015;8(1):123-150.

4. Peters BM, ME Shirtliff, MA Jabra-Rizk. Antimicrobial peptides: primeval molecules or future drugs. PLoS Pathog. 2010;6(10):1-4.

5. Hector Mendez-Figueroa; Brenna Anderson. Vaginal Innate Immunity, Alteration During Pregnancy and its Impact on Pregnancy Outcomes. Expert Rev of Obstet Gyneco. 2011;16(6):629-641.

6. Fjell CD, Hiss JA, Hancock REW, et al. Designing antimicrobial peptides: form follows function. Nature Reviews Drug Discovery. 2012;11(1):37-51

7. Maisetta G, Di Luca M, Esin S, et al. Evaluation of the inhibitory effects of human serum components on bactericidal activity of human beta defensin 3. Peptides. 2008;29(1):1-6.

8. Chang TL, Vargas J Jr. Dual role of $\alpha$-defensin-1 in anti-HIV-1 innate immunity. J Clin Invest. 2005;115(3):765-773. 
9. Quinones-Mateu ME, Lederman MM. et al. Human epithelial $\beta$-defensin 2 and 3 inhibit HIV-1 replication. AIDS. 2003;17(16):F39-F48.

10. Cole AM, Cole AL. Antimicrobial polypeptides are key anti-HIV-1 effector molecules of cervicovaginal host defense. Am J Reprod Immunol. 2008;59(1):27-34.

11. Novak RM, Donoval BA, Graham PJ, et al. Cervicovaginal levels of lactoferrin, secretory leukocyte protease inhibitor, and RANTES and the effects of coexisting vaginoses in human immunodeficiency virus (HIV)seronegative women with a high risk of heterosexual acquisition of HIV infection. Clin Vaccine Immunol. 2007;14(9):1102-1107.

12. Que ML, Andersen E, Mombelli A. Myeloid-related protein (MRP)8/14 (calprotectin) and its subunits MRP8 and MRP14 in plaque-induced early gingival inflammation. J Clin Periodontol. 2004;31(11):978-984.
13. Iqbal SM, Ball TB, Levinson $P$, et al. Elevated elafin/trappin-2 in the female genital tract is associated with protection against HIV acquisition. AIDS. 2009;23(13):1669-1677.

14. Srakaew N, Young CD, Sae-wu A, et al. Antimicrobial host defence peptide, LL-37, as a potential vaginal contraceptive. Hum Reprod. 2014;29(4):683-696.

15. Nongnuj Tanphaichitr, Nopparat Srakaew, Rhea Alonzi, et al. Potential Use of Antimicrobial Peptides as Vaginal Spermicides/Microbicides. Pharmaceuticals (Basel). 2016;9(1):1-35.

16. Antimicrobial Peptide Database (APD3). 2017. 